\title{
La reconstitution quasi exacte du grand diamant bleu de Louis XIV
}

François Farges (francois.farges@mnhn.fr)

Muséum national d'Histoire naturelle, Institut de minéralogie, physique des matériaux et cosmologie (UMR CNRS 7590), Institut Universitaire de France

Muséum national d'Histoire naturelle, IMPMC - CP 52, 61 rue Buffon, 75005 Paris

Les minéraux - et plus

particulièrement les gemmes ont été les instruments

de pouvoir privilégiés

des souverains du passé.

Entre vols, ventes et autres

aliénations, la majorité de ces

chefs-d'œuvre de l'art lapidaire

n'ont pas survécu. La science

- qu'elle soit histoire de l'art

ou physique des géomatériaux -

nous aide à les restituer

de manière rigoureuse.

Ainsi, la spectroscopie optique quantitative permet de reconstituer exactement le fabuleux diamant bleu de Louis XIV,

qui avait été volé en 1792 sans

laisser de traces évidentes.

Des nanotechnologies permettent même de recréer une réplique exacte de ce diamant perdu, une rareté naturelle.

Les termes en gras suivis d'un astérisque sont définis dans le glossaire, pp. 50-51.
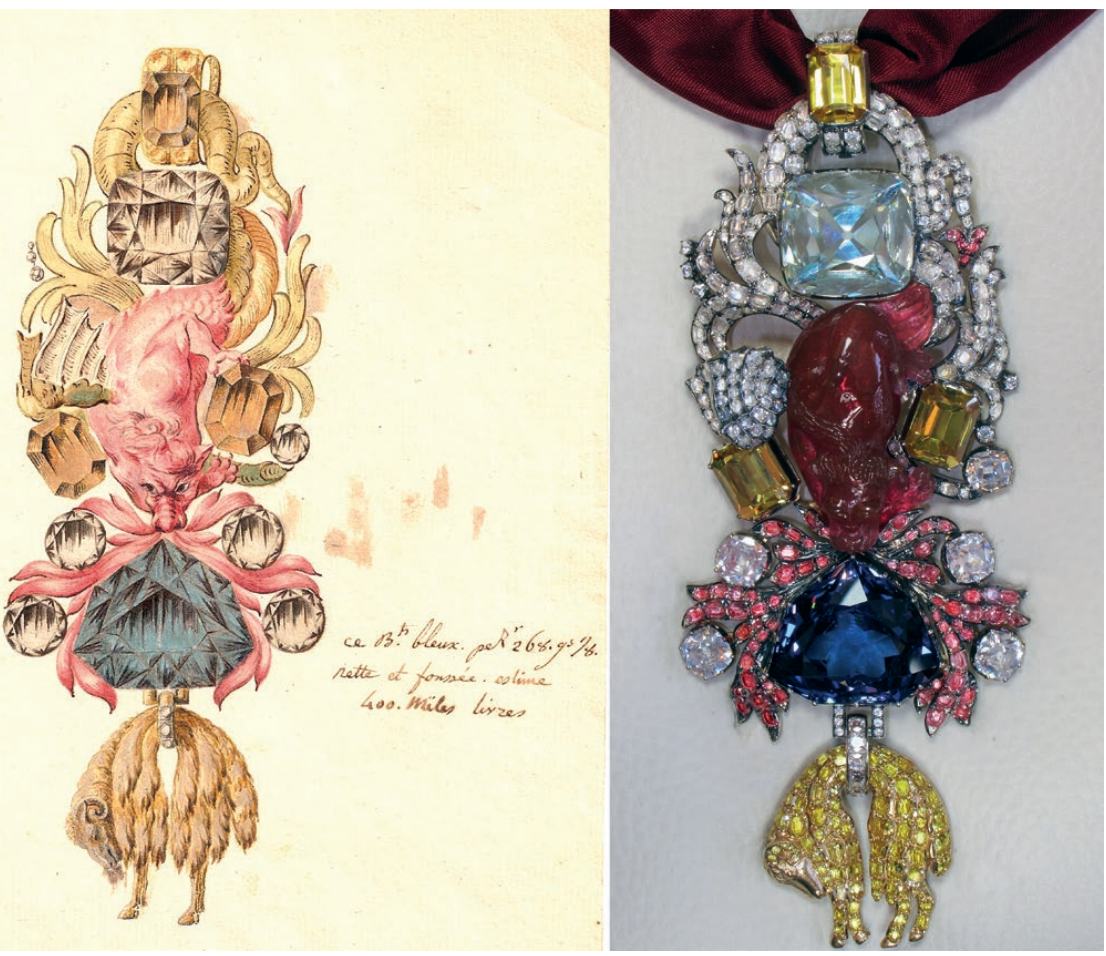

1. La Toison d'Or de Louis XV. À gauche, gravure peinte de la Toison d'Or (vers 1749), où I'on voit le grand diamant bleu de Louis XIV de forme triangulaire, que son arrière petit-fils, Louis XV, a fait ressertir dans son insigne par Jacqmin. À droite, la Toison d'Or reconstituée en gemmes de synthèse. (Collection H. Horowitz, avec permission.)

En septembre 1792, disparaissait l'un des plus précieux chefs d'œuvre : le grand insigne de la Toison d'Or* de Louis XV [1]. La parure, conçue en 1749 par Pierre-André Jacqmin (1720-1773) est l'objet fondateur de la haute joaillerie parisienne, même si d'autres pièces maitresses ponctuaient l'histoire joaillière déjà riche de la capitale française. L'insigne comportait le plus imposant des diamants bleus jamais trouvés à ce jour. Il était "gardé » par un dragon, délicatement sculpté dans un spinelle par Jacques Guay (1711-1793), favori de la favorite, la Pompadour. À travers cet insigne riche de symboles, Louis XV défie toute l'Europe par sa puissance : la Toison d'Or est source infinie de richesse et d'ardeur militaire. 
Des objets de musée aux modèles numériques
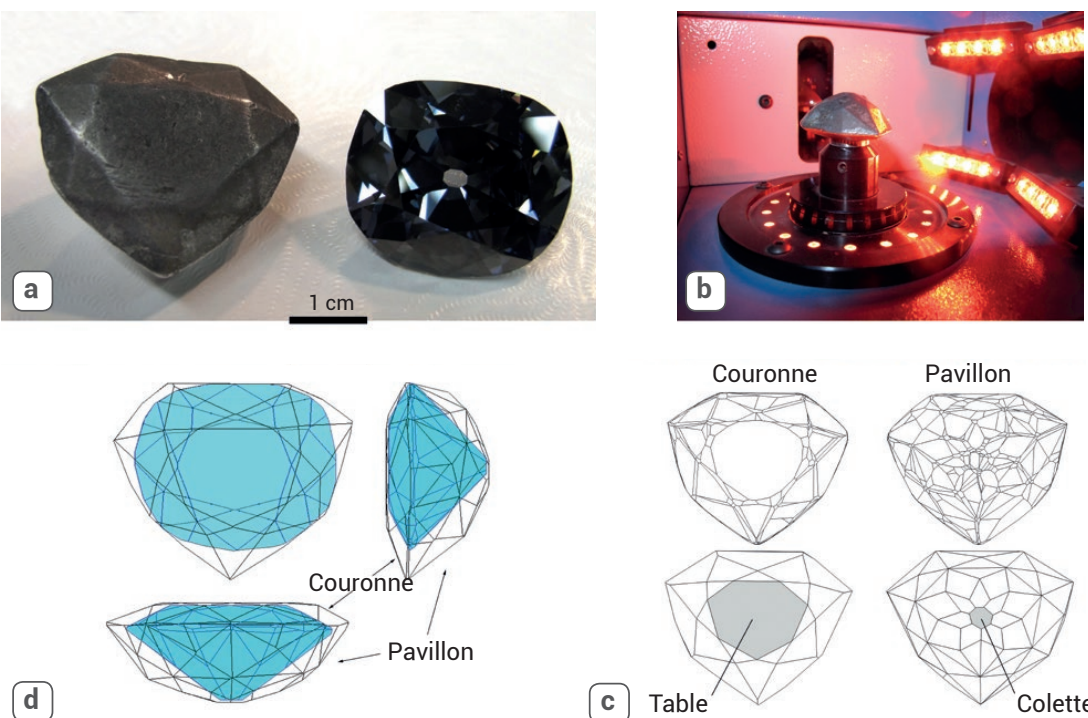

d

Les figures ci-dessus montrent la reconstitution numérique des facettes du diamant bleu et sa comparaison avec le diamant Hope.

(a) Le moulage en plomb du diamant bleu retrouvé au Muséum national d'Histoire naturelle en 2008 et le diamant Hope desserti et posé sur un carton blanc satiné et tramé. Notez comment le papier blanc se distingue parfaitement au centre du diamant Hope, là ou il est le plus épais $(12,9 \mathrm{~mm})$, alors qu'en périphérie la gemme apparait opaque, son épaisseur se réduisant à $2 \mathrm{~mm}$.

(b) Le scan du moulage permet de repérer dans l'espace la disposition des facettes polygonales réfléchissant les pinceaux lumineux.

(c) Ces polygones ont ensuite été regroupés via des algorithmes de décimation pour reconstituer les facettes effectives de la gemme, que ce soit pour le dessus (couronne*) ou le dessous (pavillon*).

(d) Ce modèle (lignes noires) est comparé à celui obtenu sur le Hope (lignes bleues), de manière à observer la correspondance entre les deux gemmes sous trois angles de vue différents.

(Images et dessins de l'auteur)

>>

Le diamant bleu est la «pièce de résistance » de cet insigne : unique de par son poids jamais égalé depuis, 69 carats ${ }^{\star}$ soit 13 grammes environ. Après sa disparition en 1792, les enquêtes de police d'alors démontrent que le joyau fut cassé et emporté par un certain Cadet Guillot Lordonné, un jeune escroc qui s'enfuit vers Londres où il sera finalement emprisonné [1]. Mais l'insigne disparait à tout jamais. Aucune gravure ni peinture fiable de ce joyau ne semble connue : la France oubliera rapidement son chef d'œuvre, jusqu'en 2006-2007, où diverses découvertes vont précipiter sa "renaissance». En 2006, une gravure inconnue de la Toison d'or, réalisée par Jacqmin, apparait à Genève (fig. 1), alors que je retrouvais au Muséum national d'Histoire naturelle l'unique moulage ${ }^{\star}$ connu du grand diamant bleu volé en 1792 [2] (encadré 1).

\section{Une enquête scientifique qui en amène d'autres}

La première enquête a consisté à départager les deux adversaires se réclamant être l'avatar du diamant bleu français. D'un côté, le clan russe qui, à travers le diamant Terestchenko de 42,92 carats, revendique la prestigieuse paternité de par sa forme ovoïde. De l'autre, le clan américain avec le diamant Hope` de 45,52 carats, conservé à la Smithsonian Institution (Washington, USA) où il est - les Français l'ignorent - l'objet le plus visité au monde avec la Joconde du Louvre.

Un scan laser de la surface du moulage en plomb a été réalisé pour obtenir un maillage ${ }^{\star} 3 \mathrm{D}$ de milliers de polygones décrivant cette surface. Des méthodes numériques comme la décimation ${ }^{\star}$ par effondrements d'arêtes ont permis de restituer le facettage d'origine de la gemme*. Ce résultat, comparé à celui obtenu pour les deux avatars actuels en compétition, est clair : le Terestchenko est trop allongé et pas assez large. Le diamant américain gagne haut la main (encadré 1). Les voleurs de 1792 ont donc sauvagement raboté les coins du diamant triangulaire français pour lui donner la forme ovoïde actuelle du Hope.

\section{La couleur}

Ensuite, se posa la question de la couleur de cette gemme mythique. La confusion règne : décrit comme "violet " dans les inventaires royaux de 1691, d'un bleu de saphir en 1787 puis bleu roi en 1813 . L'étude débuta sur son avatar actuel formellement identifié, le diamant Hope, démonté de son collier. Un aspect optique - jamais noté ni interprété auparavant fut révélateur : au centre de la gemme, où elle est la plus épaisse, l'objet se comporte comme un prisme à faces parallèles peu coloré, délimité par sa

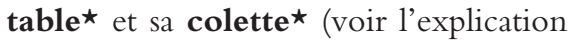
dans l'encadré 2, p. 49). En revanche, en périphérie, le diamant apparait bleu foncé alors qu'il est bien moins épais. Pour comprendre cette curieuse anomalie, une étude d'absorption optique a été entreprise sur cette gemme.

\section{L'ordinateur, décodeur du génie créateur du lapidaire de Louis XIV}

De lourds calculs ab initio de la couleur du Hope ont été entrepris pour élucider son anomalie (fig. 2). La fonction diélectrique théorique d'un diamant a été calculée en considérant un dopage au bore, présent à l'état de traces dans la structure atomique carbonée de ce minéral` et à l'origine de sa couleur bleue [3]. Nous avons ensuite résolu l'équation de Bethe-Salpeter ${ }^{\star}$ [4] qui permet de quantifier l'interaction entre la lumière et ce minéral pour en déterminer son spectre d'absorption entre les ultraviolets et l'infrarouge proche (400-900 nm). Ces travaux nous ont permis de reproduire et de comprendre l'anomalie de couleur de ce diamant. En fait, le diamant Hope n'est pas bleu foncé mais bleu pâle! Le calcul a pu montrer que son facettage particulier amplifie la couleur pâle du 
minéral au point qu'il nous apparait bleu foncé, voire ultra-marin, sauf en son centre où l'on peut voir sa vraie couleur sans les interférences dues au facettage (fig. 2).

Comme le facettage du Hope differe drastiquement de celui de son ancêtre, il nous a fallu alors recalculer la couleur effective du diamant français sur la base du moulage scanné et décimé. Ce protocole calculé (encadré 2), nous avons obtenu un diamant aux bleus centrés sur un... bleu roi! Mieux, quand nous l'avons serti d'or comme le suggère l'inventaire de 1691 des joyaux de la Couronne de France, un soleil d'or est apparu en son centre : clairement, ce « soleil royal » est l'emblème de Louis XIV et, plus généralement, de la France monarchique d'alors [2].

\section{Au-delà des calculs, la gemme renait...}

Pour nous affranchir du virtuel, nous avons reconstitué le diamant " en vrai ". Le défi est de taille : aucun autre diamant bleu de ce poids n'a jamais été retrouvé depuis 1668. Et si ce minéral avait existé, le prix en aurait été stratosphérique. Une autre piste a été considérée : la zircone cubique, $\mathrm{ZrO}_{2}$, est un oxyde de zirconium cristallisé qui possède des propriétés optiques assez proches de celles du diamant sans couter une fortune. Mais aucune zircone cubique ne possède la couleur "bleue froide" si typique des diamants bleus. Au contraire, nos essais de dopage se sont soldés par la création de répliques trop violettes. Ces zircones bleues industrielles, dopées aux terres rares donnent systématiquement une couleur trop enrichie en rouge secondaire par rapport au bleu, la couleur primaire. De plus, ces zircones sont trop teintées dans la masse, ce qui absorbe une grande quantité de la lumière réfléchie et dispersée par ce matériau. La différence avec les simulations théoriques est trop énorme.

En fait, si la zircone incolore est un bon modèle pour les diamants sans couleur, on ne reconnait pas les caractéristiques optiques d'un diamant bleu dans ces zircones dopées. Nos efforts furent sans succès pendant des années. Si la chimie ne semblait pas pouvoir répondre à ce défi, nous avons regardé du côté des " couleurs physiques ", les couleurs "photoniques " telles celles générées par les écailles de papillon ou par l'iridescence de la nacre.

\section{Principes optiques des diamants taillés} Encadré 2

a
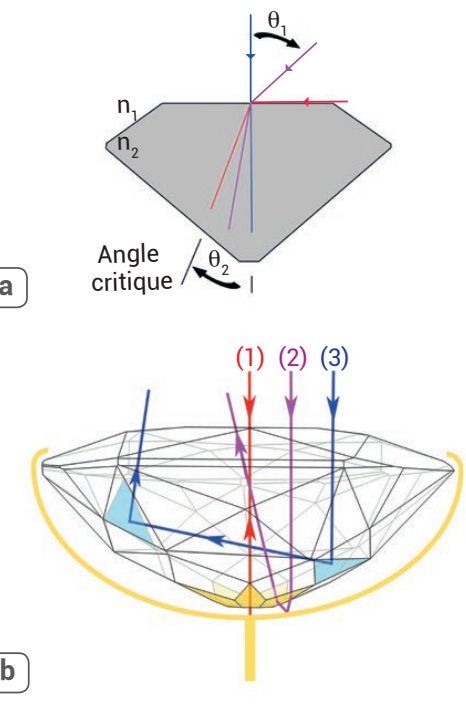

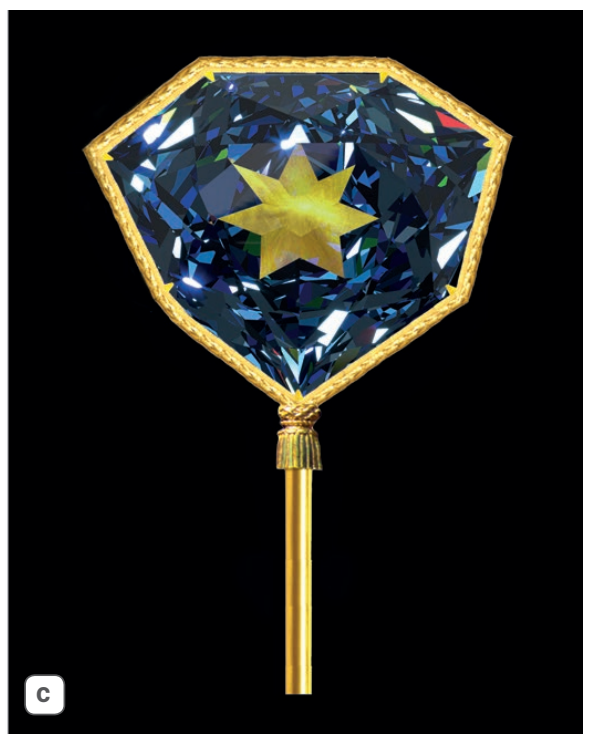

(a) Cette figure explicite la définition et la mesure de l'angle critique, $\theta_{2}$, pour un dioptre air (1) / gemme (2) d'indices de réfraction $n_{1}$ et $n_{2}$.

Pour une incidence lumineuse inférieure à cet angle, le diamant se comportera comme un dioptre transparent. Sinon, les facettes de la gemme vont se comporter comme des miroirs : la lumière sera davantage absorbée et la couleur sera perçue comme plus intense (foncée). L'angle critique vaut environ $26^{\circ}$ pour le diamant.

(b) L'application de ce concept au diamant bleu de Louis XIV serti dans une coquille d'or (vue en coupe). Le trajet de lumière (1) passe à travers le diamant, étant donné son angle d'incidence nulle par rapport à la normale de la table du diamant. II passe le dioptre diamant/or et renvoie l'image du serti-or sous-jacent. Le trajet (2) correspond à une incidence légèrement décentrée de la lumière sur le diamant. Ici aussi, l'angle d'incidence est inférieur à l'angle limite et la lumière est encore renvoyée par l'or. Quant au trajet (3), il correspond à de la lumière arrivant sur les facettes du pavillon avec un angle supérieur à l'angle critique : la lumière est alors réfléchie dans le diamant qui augmente son absorption et, par conséquent, fonce la couleur perçue par l'observateur en retour. Clairement, le lapidaire* de Louis XIV devait maitriser, sciemment ou non, les concepts fondateurs de l'optique linéaire publiés par René Descartes dans La Dioptrique, annexe du Discours de la Méthode (Leyden, 1637).

(c) La simulation infographique du diamant bleu de Louis XIV sur serti-or suit les indications de l'inventaire des diamants de la Couronne de 1691 (Archives nationales, $0^{1}, 3360$ ). Le soleil-royal central rayonne dans un univers étoilé. Les sept facettes dorées situées autour de la facette centrale (colette) symbolisent les sept planètes alors considérées (Mercure, Vénus, Terre, Mars, Jupiter et Saturne, y compris la Lune). Suivant l'inclinaison du diamant, ces facettes s'allument ou s'éteignent alors que la colette reste « toujours allumée d'or ». Nous postulons qu'elles représentent une allégorie des grâces et disgrâces du roi envers la Cour inféodée à son bon vouloir.

Cette vision héliocentriste contraste avec les dogmes platoniciens alors reconnus par l'Église, selon lesquels le Soleil est censé graviter autour de la Terre. II faudra attendre 1755 pour que le Vatican admette cette version du système solaire. Clairement, Louis XIV n'a pas attendu : dès 1664, Le traité du monde et de la lumière sera publié avec approbation royale alors que son auteur, René Descartes, prend fait et cause pour Galilée et ses écrits sur l'héliocentrisme. 


\section{Références}

1• G. Bapst, Histoire des joyaux de la Couronne de France, Hachette (1889).

2• F. Farges, « Les grands diamants de la Couronne, de François $1^{\text {er }}$ à Louis XVI ", Versalia 17 (2014) 55-78.

$3 \cdot$ E. Fritsch, "The nature of color in diamonds", dans The Nature of Diamonds (édité par G.E. Harlow), Cambridge University Press (1998), pp. 23-47.

4• F. Farges, J. Vinson, J.R. Rehr et J.E. Post, "The recovery of the French Blue diamond", Europhysics News, 43 (2012) 22-25.

5. J.E. Post, F. Farges et al., "Now there are near-perfect copies of the Hope diamond" (2017), www.smithsonianmag. com/smithsonian-institution/ now-there-are-near-perfect-copieshope-diamond-180967372 .

>>

En effet, il est possible de donner l'illusion d'une couleur en déposant des nanoparticules métalliques pulvérisées à haute température et sous ultravide sur un substrat de zircone incolore. La couleur - obtenue par diffusion de la lumière par cette couche mince de nanoparticules - est ajustable suivant les conditions opératoires (ultravide, température, nature du métal à déposer, etc.). Après deux années d'essais via la société Azotic LLC de Rochester (New York, USA), la bonne recette (un secret industriel...) a été trouvée pour fabriquer une nanocouche de cristaux métalliques aux dimensions et formes provoquant une couleur physique bleue désaturée de rouge. Ensuite, une zircone incolore taillée en forme de diamant bleu de Louis XIV a été " enrobée » à sa surface par nanodéposition sous ultravide : elle reproduit presque parfaitement la simulation théorique de l'original en diamant bleu [5]. Le même mécanisme a été appliqué au diamant Hope : le résultat est si probant qu'un gemmologue averti ne se rend pas compte de la différence entre l'original et la copie sur la base de l'observation optique (la distinction se fait sur la densité, puisque la zircone est 25\% plus dense que le diamant, soit 4,4 contre 3,5 en moyenne).

De surcroit, cette réplique sera bientôt sertie dans l'or de manière à ce que nous puissions revoir ce magnifique diamant comme le Roi-Soleil le vit jadis : un
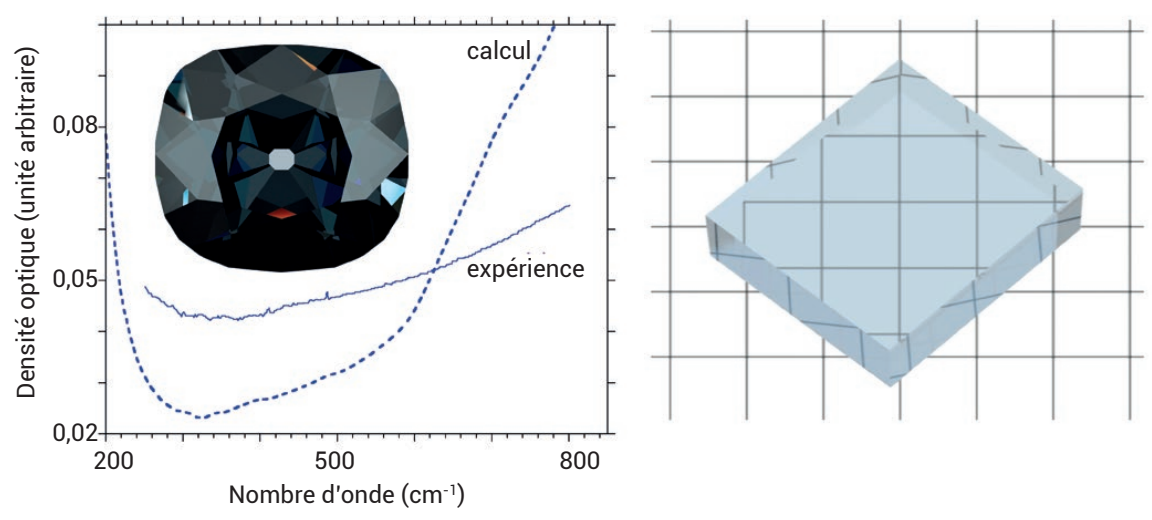

\section{2. Étude optique du diamant Hope.}

À gauche : spectres d'absorption optique du diamant Hope (trait plein), du calcul théorique d'un diamant dopé au bore (tirets) et simulation purement théorique du diamant Hope (à comparer avec l'original, représenté dans la figure (a) de l'encadré 1, p. 48).

À droite, simulation théorique d'un prisme simple taillé dans la matière minérale constituant le diamant Hope : sa couleur apparente est alors bleu pâle.

instrument politique aux couleurs du monarque et de la France. La Toison d'Or fut aussi reconstituée avec ses grands brillants ${ }^{\star}$, son spinelle et ses myriades de microdiamants, telle une micromosaïque à l'éclat incomparable que le gouaché historique peinait à traduire (fig. 1, gauche).
Ces chefs-d'œuvre retrouvés et recréés de notre histoire, le nouveau grand diamant bleu de Louis XIV ainsi que la Toison d'Or de Louis XV, n'attendent que leur mécène pour que ces répliques puissent être exposées dans un musée public français et qu'elles soient réhabilitées au sein de l'héritage créatif parisien.
: Glossaire

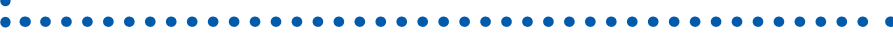

Brillant Diamant facetté d'une manière qui accentue la brillance et l'éclat. Principe mis au point probablement vers 1663.

Carat Unité de poids pour les gemmes, valant $1 / 5^{\mathrm{e}}$ de gramme, inspiré de la graine de caroubier.

Couronne Partie supérieure (avers) d'une gemme. Voir table, pavillon, colette.

Colette Facette la plus en arrière d'une gemme, généralement de petites dimensions. Elle est souvent absente dans les brillants modernes, mais commune dans les brillants anciens.

Décimation Ensemble de techniques numériques consistant à regrouper des polygones en supprimant leur arête commune. 


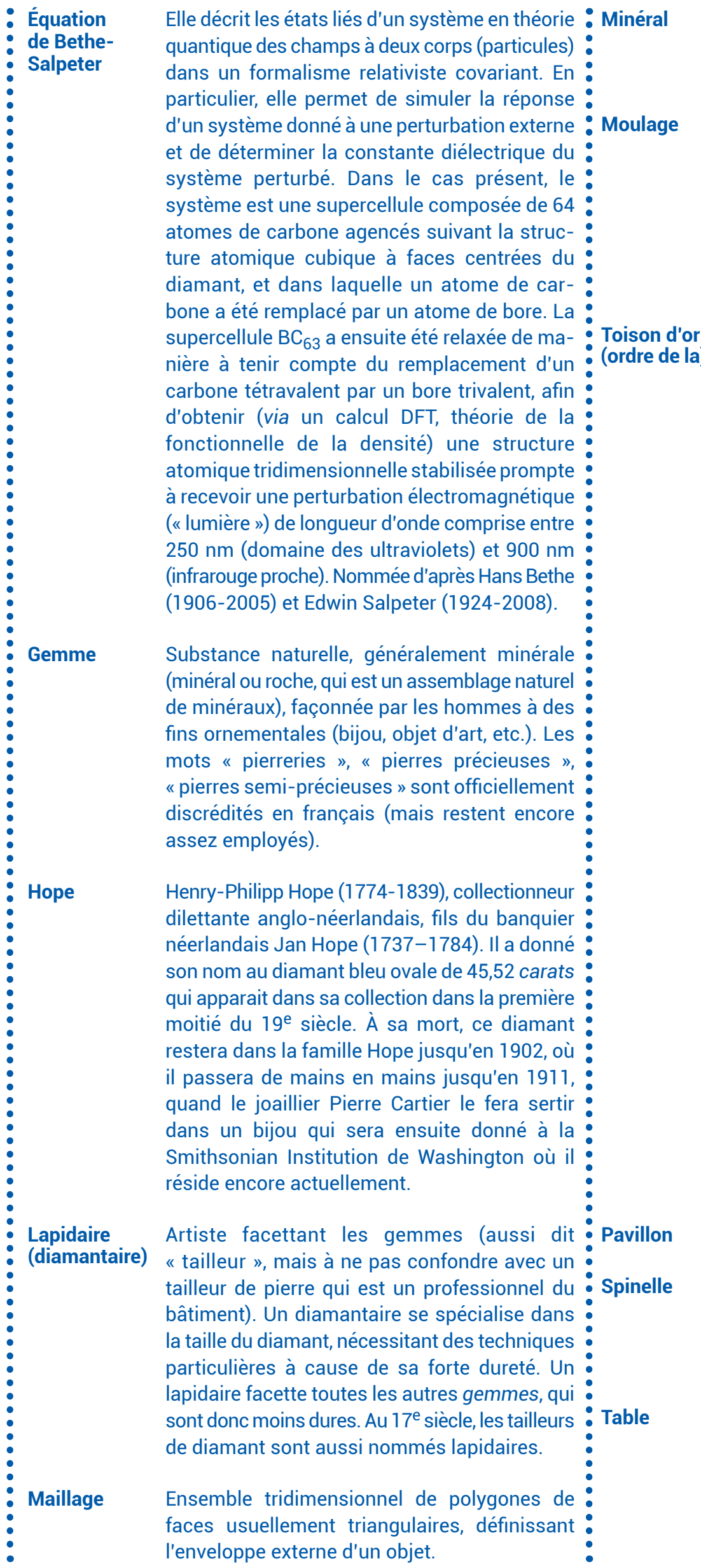

Substance naturelle d'origine (bio)géochimique, usuellement solide et cristallisée (mais pas toujours).

Empreinte d'une gemme (dans le cas présent) à des fins pédagogiques et de documentation d'un lapidaire qui ne peut garder les grandes pierres trop onéreuses, mais sur lesquelles il a investi et travaillé. Jusqu'au $19^{\mathrm{e}}$ siècle, les moulages étaient faits de plomb, puis de plâtre. De nos jours, ils sont fabriqués en résine.

Ordre de chevalerie fondé en 1430 par Philippe le Bon (1396-1467), duc de Bourgogne, pour s'assurer de la fidélité de ses chevaliers à une époque où les alliances se renversaient trop rapidement. Peu de temps auparavant, Jeanne d'Arc avait fédéré autour d'elle un grand nombre de chevaliers qui combattaient pour le roi de France. En réponse, la Bourgogne, alliée des Anglais pendant cette période de la Guerre de Cent Ans, institue cet ordre chevaleresque pour pérenniser sa force militaire autour d'une aristocratie fidélisée via un serment religieux d'allégeance irrévocable. De nos jours, l'ordre est toujours actif, notamment sa branche espagnole. L'insigne de la Toison d'or montre une " pierre à feu " (en théorie, un silex, mais souvent sublimé par un saphir ou un diamant) émettant des étincelles qui symbolisent l'ardeur guerrière du chevalier titulaire de cet ordre. La toison, à proprement parler, est suspendue tout en bas de l'insigne : elle constitue le symbole du bélier Chrysomallos, dont la précieuse pelisse apporte prospérité financière à son porteur. Dans l'insigne de Jacqmin pour Louis XV, le plus somptueux réalisé à ce jour, le joaillier a ajouté la symbolique grecque du dragon Ladon qui, selon la mythologie, veille sur la riche pelisse. Louis XV est le premier roi de France à porter cet insigne, suite à la guerre de succession d'Espagne (1701-1714) qui placera un héritier agnatique de la dynastie française des Bourbons sur le trône hispanique. L'insigne sera volé et détruit en 1792.

Partie inférieure (revers) d'une gemme.

Oxyde minéral de magnésium et d'aluminium, très prisé quand il est de qualité gemme et de toutes couleurs. La variété gemme et rouge était souvent appelée (à tort) rubis.

Grande facette constituant le dessus d'une gemme, parallèle à la colette (si présente). 